\title{
Towards a unified model for black hole X-ray binary jets
}

\author{
R. P. Fender ${ }^{1}$, T. M. Belloni², E. Gallo ${ }^{1}$ \\ ${ }^{1}$ Astronomical Institute 'Anton Pannekoek', University of Amsterdam, and Center for High Energy Astrophysics, Kruislaan 403, \\ 1098 SJ, Amsterdam, The Netherlands rpf@science.uva.nl, egallo@science.uva.nl \\ ${ }^{2}$ INAF - Osservatorio Astronomico di Brera, Via E. Bianchi 46, I-23807 Merate, Italy belloni@merate.mi.astro.it
}

5 March 2018

\begin{abstract}
We present a unified semi-quantitative model for the disc-jet coupling in black hole X-ray binary systems. In the process we have compiled observational aspects from the existing literature, as well as performing new analyses. We argue that during the rising phase of a black hole transient outburst the steady jet known to be associated with the canonical 'low/hard' state persists while the X-ray spectrum initially softens. Subsequently, the jet becomes unstable and an optically thin radio outburst is always associated with the soft X-ray peak at the end of this phase of softening. This peak corresponds to a 'soft very high state' or 'steep power law' state. Softer X-ray states are not associated with 'core' radio emission. We further demonstrate quantitatively that the transient jets associated with these optically thin events are considerably more relativistic than those in the 'low/hard' X-ray state. This in turn implies that as the disc makes its collapse inwards the jet Lorentz factor rapidly increases, resulting in an internal shock in the outflow, which is the cause of the observed optically thin radio emission. We provide simple estimates for the efficiency of such a shock in the collision of a fast jet with a previously generated outflow which is only mildly relativistic. In addition, we estimate the jet power for a number of such transient events as a function of X-ray luminosity, and find them to be comparable to an extrapolation of the functions estimated for the 'low/hard' state jets. The normalisation may be larger, however, which may suggest a contribution from some other power source such as black hole spin, for the transient jets. Finally, we attempt to fit these results together into a coherent semi-quantitative model for the disc-jet coupling in all black hole X-ray binary systems.
\end{abstract}

Key words: Accretion, accretion discs - Black hole physics - ISM: jets and outflows - Xrays: binaries

\section{INTRODUCTION}

Relativistic jets are a fundamental aspect of accretion onto black holes on all scales. They can carry away a large fraction of the available accretion power in collimated flows which later energise particles in the ambient medium. The removal of this accretion power and angular momentum must have a dramatic effect on the overall process of accretion. In their most spectacular form they are associated with supermassive black holes in active galactic nuclei (AGN), and with Gamma-Ray Bursts (GRBs), the most powerful and explosive engines in the Universe respectively. However, parallel processes, observable on humanly-accessible timescales, are occurring in the accretion onto black holes and neutron stars in binary systems within our own galaxy.

The current observational picture of X-ray binary jets is most simply put as follows: in the low/hard state, which exists typically below a few $\%$ of the Eddington luminosity $L_{\mathrm{Edd}}$ (e.g. Maccarone 2003; McClintock \& Remillard 2004) there is a 'compact' selfabsorbed jet which manifests itself as a 'flat' (spectral index $\alpha \sim 0$ where $\left.\alpha=\Delta \log S_{\nu} / \Delta \log \nu\right)$ or 'inverted' $(\alpha \geq 0)$ spectral com- ponent in the radio, millimetre and (probably) infrared bands (e.g. Fender 2001b, Corbel \& Fender 2002). The radio luminosity of these jets shows a strong, non-linear correlation with X-ray luminosity (Corbel et al. 2003; Gallo, Fender \& Pooley 2003) and has only been directly spatially resolved in the case of Cyg X-1 (Stirling et al. 2001), although the 'plateau' jet of GRS $1915+105$ is phenomenologically similar and has also been resolved (Dhawan et al. 2000; Fuchs et al. 2003). The suggestion that such steady, compact jets are produced even at very low accretion rates (Gallo, Fender \& Pooley 2003; Fender, Gallo \& Jonker 2003) has recently received support in the flat radio spectrun observed from the 'quiescent' transient V404 Cyg at an average X-ray luminosity $L_{\mathrm{X}} \sim 10^{-6} L_{\text {Edd }}$ (Gallo, Fender \& Hynes 2004). During steady 'soft' X-ray states the radio emission, and probably therefore jet production, is strongly suppressed (Tanabaum et al. 1972; Fender et al. 1999b; Corbel et al. 2001; Gallo, Fender \& Pooley 2003).

Additionally there are bright events associated with transient outbursts and state transitions (of which more later), which are often directly resolved into components displaying relativistic motions away from the binary core (e.g. Mirabel \& Rodriguez 1994; 
Hjellming \& Rupen 1995; Fender et al. 1999) not only in the radio but also - at least once - in the X-ray band (Corbel et al. 2002). These events typically display optically thin (synchrotron) radio spectra $(\alpha \leq-0.5)$. Both kinds of jets are clearly very powerful and coupled to the accretion process. See Mirabel \& Rodriguez (1999) and Fender (2004) for a more thorough review of the observational properties of X-ray binary jets.

In this paper we attempt to pin down as accurately as possible the moment at which the major radio outburst occurred and relate this to the X-ray state at the time. We subsequently compare this with the X-ray state corresponding to the lower-luminosity steady jets, to the evolution of transient outbursts, and to the velocity and power associated with each 'type' of jet, in order to draw up a framework for a unified model of black hole X-ray binary jet production.

Several black hole systems are investigated in this paper, and in addition we compare these with the neutron star systems Cir X1 and Sco X-1. The data relating to the radio flares, jet Lorentz factors (if measured), corresponding X-ray luminosities, estimated distances and masses, are summarised in Table 1.

\section{THE SAMPLE: FOUR BLACK HOLES UNDERGOING JET FORMATION}

Is there a signature in the X-ray light curve of an outbursting source which indicates when the relativistic jet is launched? In the following we investigate radio and X-ray light curves of four black hole binaries - GRS 1915+105, GX 339-4, XTE J1859+226 and XTE J1550-564 - undergoing state transitions in order to investigate this point.

\subsection{X-ray Data analysis}

For XTE J1550-564, XTE J1859+226 and GX 339-4, we extracted the background-subtracted PCA count rate, using PCU2 only, for the each available RXTE observation relative to the first part of the outburst considered. For each observation, we also produced an Xray color (or hardness ratio) by dividing the background-subtracted count rates in the $6.3-10.5 \mathrm{keV}$ band by those in the $3.8-6.3 \mathrm{keV}$ band. For GRS 1915+105, given the much shorted time scales involved, we analyzed a single observation (see Sect. 2.2.1), producing a PCA light curve at 1 second resolution from all PCUs summed together, and an X-ray color curve at the same time resolution. The energy bands used for the color, 15.2-42.3 keV and 2.1-5.9 keV were different. The reason for which is that the thermal disc component in GRS $1915+105$ is considerably higher than in other systems (see Fender \& Belloni 2004): using the same energy bands results in the harder $\mathrm{C}$ state having a softer color than the softer $\mathrm{B}$ state. The use of a harder energy band for the numerator ensures that the thermal disc component does not strongly contaminate it.

In order to estimate in a homogeneous way the source luminosity at peak of the outburst for XTE J1550-564, XTE J1859+226 and GX 339-4, we extracted RXTE PCA+HEXTE spectra from the public archive corresponding to the peak flux in the PCA band. Spectra were created from PCU2 data (3-25 keV) for the PCA and from cluster A data (20-200 keV) for HEXTE, using FTOOLS 5.3. The spectra were corrected for background and dead-time effects. A $0.6 \%$ systematic error was added to the PCA to account for residual calibration effects. We fitted the spectra with the standard phenomenological model for these systems, consisting of a cutoff power-law (cutoff), a disk blackbody (di skbb), a Gaussian emission line between 6 and $7 \mathrm{keV}$, all modified by interstellar absorption. The actual models might be more complex, but we are interested in the determination of the flux only, so that the presence of additional components like iron absorption edges does not change significantly our results. The reduced $\chi^{2}$ values were between 1.3 and 1.5. From the best fit models for each source, in order to estimate the bolometric flux, we computed the unabsorbed flux of the disc blackbody component in the $0.001-100 \mathrm{keV}$ range and that of the power-law component in the $2-100 \mathrm{keV}$ range. For GRS 1915+105, given the much shorter time scales involved for the short oscillations, we applied the procedure outlined above to the RXTE/PCA+HEXTE data of the observation of 1997 October 25 , the last one before the launch of the major jet observed with MERLIN (Fender et al. 1999). In order to approximate the flux evolution from the count rate light curves, we applied to all preceding and following observations the count rate to flux conversion factor obtained from the peak. A comparison with published flux values for XTE J1550-564 (Sobczak et al. 2000) this proved to be a reasonable approximation for our purposes.

Much of the following discussion is based upon the association of radio emission with the X-ray 'states' of different sources. We will use the following abbreviations throughout the paper: the 'low/hard' state as 'LS'; the 'high/soft' state as 'HS', and the 'very high' or 'intermediate' states as 'VHS/IS'. In fact, as we shall discuss further (see also Belloni et al. 1994; Homan \& Belloni 2004), the VHS/IS is not a single state but has both 'hard' (as used to describe the X-ray spectrum) and 'soft' varieties.

Note that recently McClintock \& Remillard (2004) have proposed a modification to this classification scheme in which the LS is referred to as the 'hard' state and the HS as the 'thermal dominated state', while revised definitions are introduced for the VHS/IS states. While we retain the 'classical' definitions of states, we discuss later on how these correspond to the revised state definitions of McClintock \& Remillard.

\subsection{Individual sources}

\subsubsection{GRS $1915+105$}

GRS 1915+105 has long been a key source in our understanding of the disc-jet coupling in X-ray binary systems (see Fender \& Belloni 2004 and references therein). In the context of this study it is interesting because its X-ray state never seems to reach the 'canonical' LS or HS but instead switches between 'hard' (state 'C' of Belloni et al. 2000) and 'soft' (states 'A' and 'B') VHS/IS states (see also Reig et al. 2003). It has clearly been established in this source that phases of hard (C) X-ray emission lasting more than a few hundreds of seconds are associated with radio events, and that when the source is only in soft $(\mathrm{A}, \mathrm{B})$ states there is no radio emission (Klein-Wolt et al. 2002). In the context of this work its importance is therefore in establishing that state changes 'within' the VHS/IS can produce radio outbursts without requiring any 'contact' with the canonical LS or HS. Fig 1(a) presents the lightcurve of a typical 'oscillation' event in which the source makes a transition from state $\mathrm{C}$ to state $\mathrm{A} / \mathrm{B}$. The data correspond to the RXTE observation of 1999 June 14 (class $\nu$ ), time zero is 01:00:40 UT.These oscillation events can occur in very long sequences and are generally associated with sequences of synchrotron oscillations which are almost certainly from the jets (e.g. Klein-Wolt et al. 2002). 

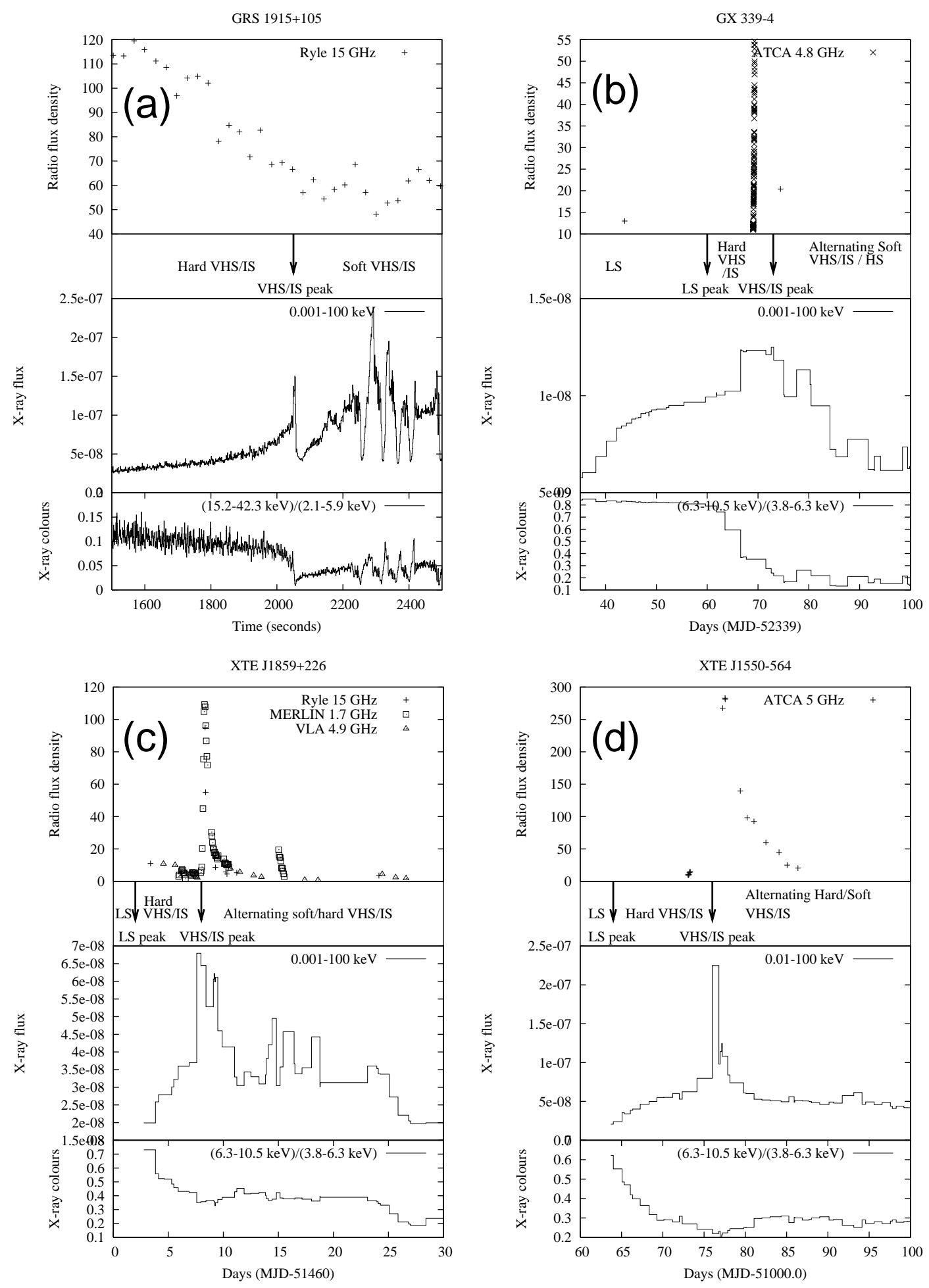

Figure 1. Radio and X-ray light curves, $\mathrm{X}$-ray colours and X-ray state classifications during periods around transient jet formation, for four black hole (candidate) X-ray binaries. In GRS 1915+105 the canonical LS or HS are never reached; in GX 339-4, XTE J1859+226 and XTE J1550-564 the delay between the canonical LS peak and subsequent VHS/IS peak ranges from a few days to two weeks. Nevertheless, in all four cases the radio flare occurs at the time of the VHS peak, indicating a clear association between this, and not the previous LS, and the major ejection. The units of the X-ray flux are erg s ${ }^{-1}$ $\mathrm{cm}^{-2}$. 


\begin{tabular}{cccccccccc}
\hline Source & $\begin{array}{c}d \\
(\mathrm{kpc})\end{array}$ & $\begin{array}{c}M \\
M_{\odot}\end{array}$ & $\begin{array}{c}\text { Date } \\
(\mathrm{MJD})\end{array}$ & $\begin{array}{c}\Delta t \\
(\mathrm{sec})\end{array}$ & $\begin{array}{c}S_{5 \mathrm{GHz}} \\
(\mathrm{mJy})\end{array}$ & $\Gamma$ & $\begin{array}{c}L_{\mathrm{J}} \\
(\mathrm{Edd})\end{array}$ & $\begin{array}{c}L_{\mathrm{X}, \mathrm{VHS}} \\
(\mathrm{Edd})\end{array}$ & Ref \\
\hline GRS 1915+105 (flare) & 11 & 14 & 50750 & 43200 & 320 & $\leq 1.4$ & 0.6 & 1.1 & F99 \\
GRS 1915+105 (osc.) & 11 & 14 & many & 300 & 50 & $\geq 2$ & 0.05 & 1.1 & F99 \\
GROJ1655-40 & 3.5 & 7 & 49580 & 43200 & 2000 & $\geq 1.7$ & 1.0 & 0.1 & HR95 \\
XTE J1859+226 & 6 & 7 & 51467 & 21600 & 50 & $?$ & 0.2 & 0.2 & B02 \\
XTE J1550-564 & 6 & 9 & 51077 & 43200 & 130 & $\geq 2$ & 0.3 & 0.5 & W02 \\
GX 339-4 & 8 & 7 & 52408 & 19800 & 55 & $\geq 2.3$ & 0.3 & 0.07 & G04 \\
V4641 Sgr & 8 & 9 & 51437 & 43200 & 420 & $\geq 9.5$ & 0.8 & 4 & Hj01, Or01 \\
Cyg X-1 & 2.5 & 10 & 53055 & 2000 & 50 & $?$ & 0.02 & $\sim 0.05$ & P04 \\
XTEJ1748-288 & 8.5 & 7 & 50980 & 172800 & 530 & $\geq 2.7$ & 1.9 & $\sim 0.1$ & B04 \\
\hline Cir X-1 & 6 & 1.4 & 51837 & 43200 & 20 & $\geq 15$ & 0.6 & $\sim 0.7$ & F04 \\
Sco X-1 & 2 & 1.4 & many & 3600 & 15 & $\geq 3.2$ & 0.02 & $\sim 1$ & F02 \\
\hline
\end{tabular}

Table 1. Parameters for the X-ray binary systems and selected jet events discussed in this paper; the last two sources contain accreting neutron stars, the rest black hole candidates. The first three columns give the source name, distance and mass of the accretor. Columns 4-9 give the date, rise time, peak radio flux, constraints on bulk Lorent factor, estimated jet power and corresponding estimated X-ray power for jet production events. The final column gives references $(\mathrm{F} 99=$ Fender et al. 1999; HR95 = Hjellming \& Rupen 1995; B02 = Brocksopp et al. 2002; W02 = Wu et al. 2002; G04 = Gallo et al. $2004 ;$ Hj01 = Hjellming et al. 2001; Or01 = Orosz et al. 2001; P04 = Pooley 2004; B04 = Brocksopp et al. (in prep); F04 = Fender et al. 2004; F02 = Fomalont et al. 2001a,b).

\subsubsection{GX 339-4}

GX 339-4 is also a key source in our understanding of the discjet coupling in X-ray binaries (Fender et al. 1999b; Corbel et al. 2000, 2003; Gallo et al. 2003; Belloni et al. 2004), albeit one which varies on timescales considerably longer than in GRS 1915+105. Recently, a clear bright optically thin radio flare was observed from this source, and subsequently found to be associated with a relativistic ejection event (Gallo et al. 2004). The light curve, corresponding to the first part of the 2002/2003 outburst (Belloni et al. 2004), presented in Fig 1(b) shows many similarities with that of GRS 1915+105 (Fig 1(a)) in the X-ray band, with a rising hard (in this case the canonical LS) state softening shortly before a soft VHS/IS peak. Subsequently both sources show a drop in the X-ray flux and then a slow recovery to even higher levels. Compared to GRS 1915+105 we see that the softening of the X-ray state began a few days before the radio flare, which seems to correspond to the VHS/IS peak near the end of the softening. However, the key thing we learn from GX 339-4 compared to GRS $1915+105$ is that the major radio event is also associated with the state transition, something which is not unambiguous in GRS $1915+105$. In this context it is interesting to note that the rise and decay timescales of the radio oscillation events in GRS 1915+105 are comparable to the durations of both the hard VHS/IS and soft VHS/IS states (since the source is in general 'oscillating' between the two). However, in GX 339-4 we see that this is clearly not the case, and that the transient radio event is associated with a specific and very limited instant in time.

\subsubsection{XTE J1859+226}

XTE J1859+226 (Fig 1(c)) is a more 'traditional' transient than either GRS $1915+105$ or GX 339-4, and underwent a bright outburst in 1999 (Wood et al. 1999; Markwardt et al. 1999; Brocksopp et al. 2002; Casella et al. 2004). It showed an initial LS peak during the rise to outburst, which preceded the subsequent soft peak by several days. This allows us to separate the LS and VHS/IS peaks - which was impossible for GRS 1915+105 and still difficult for GX 339-4 - and identify which is associated with the radio event. The radio data used in Fig 1(c) are from Brocksopp et al. (2002).

This source peaked in hard X-rays (see BATSE light curve in Brocksopp et al. 2002) about a week before the soft peak. The $\mathrm{X}$-ray state between the two peaks can be formally described as a 'hard VHS/IS' which is gradually softening. The optically thin radio event is clearly associated not with the peak of the LS, after which the X-ray spectrum starts to soften, but with the VHS/IS peak, which seems to occur just at the end of the spectral transition. Note also that some radio emission persists after the LS peak, into the VHS/IS (further discussion below).

\subsubsection{XTE J1550-564}

XTE J1550-564 (Fig 1(d)) is another bright transient which has undergone several outbursts in recent years. The data analysed here correspond to the brightest one, in 1998/1999 (see Sobczak et al. 2000; Homan et al. 2001; Remillard et al. 2002). This outburst was associated with a very strong optically thin radio event subsequently resolved into a radio (Hannikainen et al. 2001) and, most spectacularly, an X-ray jet moving relativistically (Corbel et al. 2002). The radio data plotted in Fig 1(d) are from Wu et al. (2002) and Hannikainen et al. (2004).

As in XTE J1859+226, the LS and VHS/IS peaks are clearly separated in time, in this case by approximately two weeks. Cursory inspection of Fig 1(d) clearly indicates that the optically thin jet is launched at the time of the VHS/IS peak, which occurs, again, just at the end of the X-ray spectral transition. Once more, as in XTE $\mathrm{J} 1859+226$, we also note low-level radio emission between the LS and VHS/IS peaks.

\section{JETS AS A FUNCTION OF X-RAY STATE: NEW PERSPECTIVES}

Based upon the investigation we have performed, we are better able to associate the characteristics of the radio emission as a function of X-ray state, and therefore to probe the details of the jet:disc coupling. While the previously-established pattern of:

- $\mathrm{LS}=$ steady jet

- $\mathrm{HS}=$ no jet 

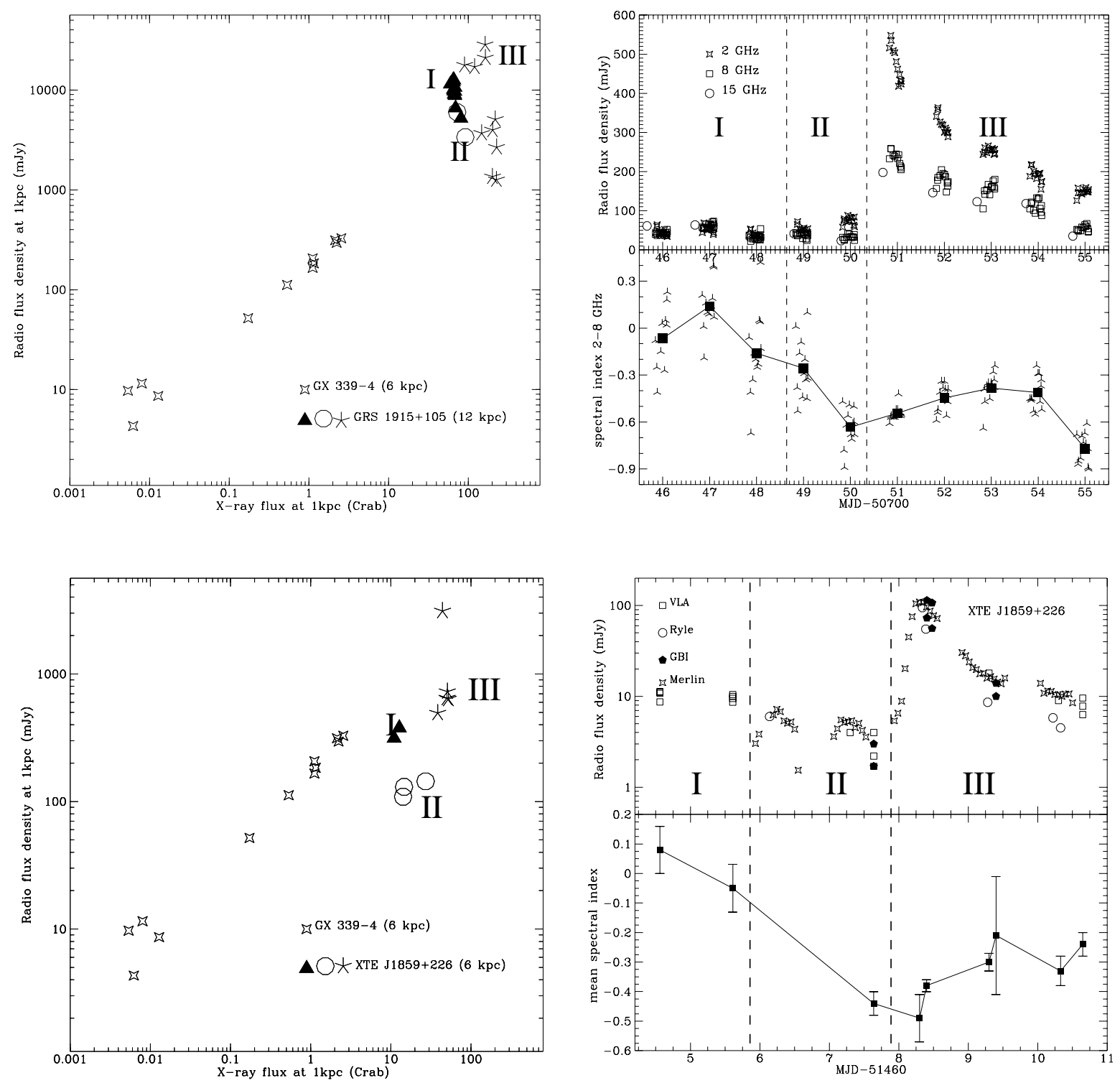

Figure 2. Behaviour of radio emission immediately prior to optically thin flare events. The top panel shows data for the GRS $1915+105$ plateau reported in detail in Fender et al. (1999); the lower panel the outburst of XTE J1859+226 reported in Brocksopp et al. (2002) and also our Fig 1. The right-hand panels show in detail the radio light curves and fitted spectral indices over this period, and are separated for each source into three phases. The location of these phases in the radio luminosity : X-ray luminosity $\left(L_{\mathrm{radio}}: L_{\mathrm{X}}\right)$ plane (Gallo, Fender \& Pooley 2003) is indicated in the left-hand panels (the X-ray luminosity is calculated from the RXTE ASM, as in Gallo, Fender \& Pooley 2003, and is therefore much less accurate than the fluxes presented in Fig 1). Initially, while still in a 'hard' VHS/IS, both sources display optically thick radio emission which lies close in the $\left(L_{\mathrm{radio}}: L_{\mathrm{X}}\right)$ plane to the mean relation for LS BH XRBs, as marked out by the data for GX 339-4 (Gallo, Fender \& Pooley 2003). Subsequently, the radio emission seems to become more erratic and occasionally optically thin.

remains valid, additional information has clearly come to light about the details of jet formation in the VHS/IS during transient outbursts.

\subsection{Persistence of the steady jet in the 'hard' VHS/IS}

It has by now been established for several years that the canonical LS is associated with persistent flat- or inverted-spectrum radio emission which probably arises in a self-absorbed, self-similar outflow (Fender 2001). This radio emission is correlated with the
X-ray emission as $L_{\text {radio }} \propto L_{\mathrm{X}}^{b}$ where $b \sim 0.7$ with a small apparent range in normalisations for several different systems (Corbel et al. 2003; Gallo, Fender \& Pooley 2003; see also Fender, Gallo \& Jonker 2003 and Jonker et al. 2004 for further discussions and implications).

While the HS was known to be associated with a dramatic decrease in the radio emission (e.g. Tananbaum et al. 1972; Fender et al. 1999b; Gallo, Fender \& Pooley 2003) it was not well known how the radio emission behaved in the VHS/IS. Fender (2001a) suggested, based upon GRS 1915+105, that the VHS/IS was associated with unstable, discrete ejection events. Corbel et al. (2001) 


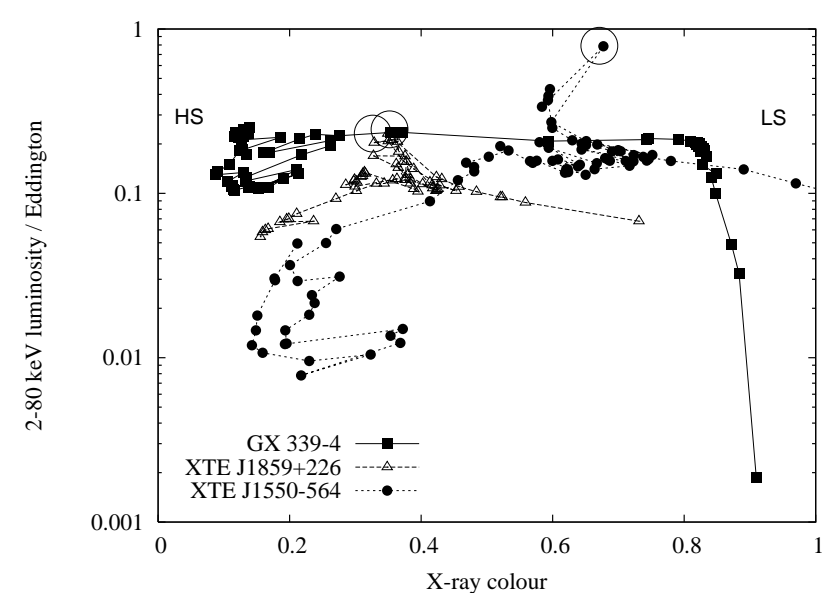

Figure 3. Combined X-ray hardness-intensity diagram (HID) for GX 3394, XTE J1859+226 and XTE J1550-564. The X-ray fluxes plotted in Fig 1(b-d) have been scaled to Eddington-ratioed luminosities using the distance and mass estimates given in table 1. Note that ejections in GX 339-4 and XTE J1859+226 occur at almost exactly the same colour and X-ray luminosity. Most of the data points correspond to varying degrees of the VHS/IS, and not the canonical LS (to the right) or HS (to the left).

reported that the radio emission from XTE J1550-564, eleven days after a transition to the VHS/IS, was reduced ('quenched') by a factor of 50 compared to the previous LS.

However, it is clear from Fig 1 that some radio emission persists beyond the end of the canonical LS, before the outburst. Some of the best data available are those of XTE J1859+226 (Brocksopp et al. 2002), which are plotted in Fig 1(c) and Fig 2. For at least three days following the LS peak, while the X-ray spectrum is softening, the radio emission is persistent with an approximately flat spectrum. Furthermore, the radio luminosity remains consistent with the universal relation of Gallo, Fender \& Pooley (2003); this is demonstrated in the left panels of Fig 2. In fact, inspecting Fig 3 of Gallo, Fender \& Pooley (2003) we can see that in the case of Cyg X-1 the turnover in the radio emission occurs after there has already been some softening of the X-ray emission, implying also that the compact jet persists into the 'hard VHS/IS'. Based on a recent study of XTE J1650-500, Corbel et al. (2004) have also concluded that the steady jet may persist beyond the initial softening of the canonical LS. The importance of this is that there is clearly not a one-to-one relation between the behaviour of the radio emission and the X-ray states as currently defined (this will be discussed more later).

\subsection{Changes in the jet radio spectrum prior to the outburst}

Following the persistence of the steady LS-like radio emission into the hard VHS/IS, the data indicate that a change in the radio emission does occur prior to the radio flare. In brief, it appears that the radio emission starts to become more variable, with a peaked or (more) optically thin spectrum shortly before the radio flare. We present the evidence for this from the four key sources under study here:

\subsubsection{GRS $1915+105$}

Given that in GRS $1915+105$ we (i) are typically observing radio emission from the last state transition, (ii) the transition period between the 'hard' and 'soft' VHS/IS is very rapid, it is not possible to investigate this effect for the oscillation-type events in this source. However, the effect is seen in the monitoring of the plateau state and subsequent radio flares as reported in Fender et al. (1999a). As noted in that work (see also discussion in Klein-Wolt et al. 2002) major radio ejections (which were directly resolved) followed the plateau. Thus, as in the other sources, a transition from the 'hard' to the 'soft' VHS/IS (as GRS 1915+105 rarely, if ever, reaches the canonical LS or HS) resulted in a major ejection. However, the data also reveal that the radio spectrum started to change prior to the first major ejection event. This is presented in Fig 2.

In fact the radio spectrum is already 'optically thin' a couple of days before the flux starts rising, reaching a mean value of -0.6 on MJD 50750, the day before the flare. The change in the radio spectrum occurs around the time that the spectrum starts to soften dramatically towards the 'soft' VHS/IS.

\subsubsection{GX 339-4}

The detailed radio light curve of GX 339-4 around the time of the major radio flare is reported in Gallo et al. (2004). During the first 2 hours of observations on MJD 52408, immediately prior to the rise of the radio flare, the spectral index seems to vary from flat to more optically thin $(\alpha \sim-0.2)$ and back to flat. Even though the error bars are relatively large, this is consistent with rapid variability of the radio spectrum outflow structure prior to the radio flare.

\subsubsection{XTE J1859+226}

In this source, over the 4 days prior to the flare on MJD 51467.5, the radio spectrum changes from flat $(0.037 \geq \alpha \geq-0.030)$ to more optically thin $(\alpha=-0.237$ on $\mathrm{MJD}=51467)$; see Fig 2 (lower panels). On this date, the radio spectrum is measured over seven frequencies: fig. 5 of Brocksopp et al. (2001) shows that the actual spectrum, even though overall optically thin, is peaked around 3 $\mathrm{GHz}$ (i.e. flat-inverted till $3 \mathrm{GHz}$ and optically thin above), indicating that a structural change in the outflow has occurred prior to the flare.

\subsubsection{XTE J1550-564}

The radio coverage of the outburst of XTE J1550-564 as presented in Fig 1(d) is reported in Wu et al. (2002). However, further measurements presented in Hannikainen et al. (2004) indicate that on MJD 51073, some 4.5 days prior to the flare, there was rapid spectral variability. This included a transition from a peaked to an optically thin spectrum, and back to a peaked spectrum again, on a timescale of less than half a day.

\subsection{Association of the outburst with the soft VHS/IS peak}

Many - perhaps all - sources which undergo an X-ray outburst have a bright hard state during the rising phase (e.g. Brocksopp et al. 2002; Maccarone \& Coppi 2003; Yu et al. 2003; Yu, van der Klis \& Fender 2004). We know that hard X-ray states correspond to phases of powerful jet production, and that the relativistic ejections tend to occur around the time of X-ray state transitions, and that soft states do not seem to produce jets (see Fender 2004 for a review). 
What was difficult to tell from investigation of the moststudied sources like GRS $1915+105$ and GX 339-4, was to what part of the state transition sequence the optically thin jet formation corresponded. The above examples appear to give a clear answer - the optically thin radio jet is produced at the VHS/IS peak, which occurs at or near the end of the X-ray spectral softening. We note that this is consistent with the suggestion of Mirabel et al. (1998) that the 'spike' in the X-ray light curve of GRS 1915+105 corresponded to the trigger point for the optically thin radio event although, as we have seen, in the case of GRS 1915+105 there are many ambiguities which cannot be resolved by studying this source in isolation.

Figure 3 presents the hardness-intensity diagrams (HIDs) for the three of the four sources presented in Fig 1, with the point corresponding most clearly to the time of the major radio flare indicated with a circle. In these HIDs the canonical LS corresponds to a nearly vertical branch on the right hand side of the diagram, seen here only for GX 339-4; the horizontal right-to-left motion exhibited by these sources is characteristic of a transition from a 'hard' to a 'soft' VHS/IS (Belloni 2004; Belloni et al. 2004; Homan \& Belloni 2004). Furthermore, the X-ray intensity has been converted to an Eddington-fraction luminosity, based upon the distance and mass estimates given in table 1 . It is clear that in all cases the radio flare occurs when the sources are in the VHS/IS, having significantly softened from the LS prior to the event, and with a X-ray luminosity in the range 10-100\% Eddington.

It is worth revisiting the result of Corbel et al. (2001) in which XTE J1550-564 was found to be radio-quiet while in the VHS/IS. An inspection of the X-ray data, indicates the following: the first radio observation, which resulted in an optically thin detection, was made within a day or two of the soft VHS/IS peak. The second observation, resulting in an upper limit only, was several days late, in the middle of the soft VHS/IS phase. Thus these observations are consistent with the picture presented here, namely that the optically thin radio flare occurs at the soft VHS/IS peak, and in the subsequent soft VHS/IS phase the core radio emission is suppressed.

Corbel et al. (2004) report a detailed study of the radio emission from the black hole transient XTE J1650-500 (plus a phenomenological comparison with XTE J1859+226 and XTE J1550564) in which the canonical LS and HS, as well as various 'degrees' of VHS/IS were observed. Specifically they conclude that the steady LS jet may persist into the 'hard' VHS/IS, and that the optically thin radio flare is associated with the soft VHS/IS peak (although they use the state definitions of McClintock \& Remillard 2004). These results seem to be consistent with the conclusions we have drawn (more quantitatively) about the association of X-ray states and jet production.

\subsection{The variation of accretion disc radius with state}

It is widely accepted that a geometrically thin, optically thick, accretion disc extends close to the black hole in 'soft' X-ray states and is 'truncated' at larger radii in 'harder' X-ray states (e.g. Esin, McClintock \& Narayan 1997; McClintock \& Remillard 2004 and references therein). While the absolute values of the radii obtained from X-ray spectral fits may be severely underestimated (e.g. Merloni, Fabian \& Ross 2000), large changes in the fitted radii are likely to be due to real changes in the location of the brightest $\mathrm{X}$ ray emitting region. Specifically, for the four sources under detailed consideration here:

\begin{tabular}{ccc}
\hline $\begin{array}{c}\text { 'Classical' } \\
\text { states }\end{array}$ & $\begin{array}{c}\text { McClintock \& } \\
\text { Remillard }\end{array}$ & Radio properties \\
\hline Low/Hard state (LS) & Hard state & steady jet \\
'Hard' VHS/IS & Intermediate state & steady jet \\
radio flare \\
'Hard' $\rightarrow$ 'soft' VHS/IS & $\begin{array}{c}\text { Intermediate } \rightarrow \text { SPL } \\
\text { 'Soft' VHS/IS }\end{array}$ & $\begin{array}{c}\text { Intermediate / SPL } \\
\text { Ther }\end{array}$ \\
High/Soft state (HS) & Thermal dominant & no jet \\
\hline
\end{tabular}

Table 2. Comparison of the 'classical' X-ray states, and those of McClintock \& Remillard (2004), with the radio properties as discussed in detail in the text. 'VHS/IS' corresponds to 'Very High State/Intermediate State' and 'SPL' stands for 'Steep Power Law'.

\subsubsection{GRS $1915+105$}

The 'dip-flare' cycles of GRS 1915+105, such as those presented in Fig 1(a), are well known to be associated with apparent changes in the fitted accretion disc radius (e.g. Belloni et al. 1997; Fender \& Belloni 2004 and references therein). During the 'soft VHS/IS' (states A/B) the fitted inner disc radius reaches a stable, low, value and is considerably larger in the 'hard VHS' (state $\mathrm{C}$ ).

\subsubsection{GX 339-4}

Spectral fits over the period focussed on in Fig 1(b) indicate a fitted inner disc radius which decreased dramatically at the point of spectral softening (Zdziarski et al. 2004; Nespoli 2004). This low value of the fitted inner radius remained stable for an extended period ( $>100$ days) until the return to the canonical LS.

\subsubsection{XTE J1859+226}

To our knowledge, detailed spectral fits over the entire outburst of XTE J1859+226 have not been published. However, both the general X-ray spectral and timing evolution and the preliminary fits reported by Markwardt (2001) indicate a similar pattern to other Xray binaries. Note that Hynes et al. (2002) discuss the post-outburst evolution of the accretion disc in XTE J1859+226 but note that the absolute value of the inner disc radius cannot be well constrained and so it is hard to use their results to compare with earlier in the outburst.

\subsubsection{XTE J1550-564}

Sobczak et al. (2000) fitted disc radii to over 200 spectra of XTE J1550-564 during the entire 1998-1999 outburst. They found that at the peak of the outburst ('soft VHS') the fitted disc radii varied a lot but were in general very (unrealistically) small. Subsequently in the canonical HS state the disc radius remained relatively small and stable over $\sim 100$ days.

\subsection{The alternative state definitions of McClintock \& Remillard}

We can summarise these connections between X-ray state and radio emission within the framework of the revised definitions of McClintock \& Remillard (2004). This is presented in Table 2.

Two significant points are worth noting: 
(i) In the framework of McClintock \& Remillard, it may be exactly at the point of the transition to the SPL that corresponds to the radio ejection event.

(ii) In the same framework, there appear to be both 'jet on' and 'jet off' phases associated with the same state 'intermediate' label.

Point (i), above, was already suggested by McClintock \& Remillard (2004) has been noted as likely also by Corbel et al. (2004). However, considering point (ii), it appears that the new definitions have both advantages and disadvantages.

\section{INCREASING VELOCITY AS A FUNCTION OF X-RAY LUMINOSITY AT LAUNCH}

A further key component for the model towards which we are progressing is the variation of jet velocity with X-ray luminosity / state. The empirical evidence clearly points to an increase of jet velocity with X-ray luminosity, at least in the sense of a step up from mildly relativistic velocities in the LS to significantly relativistic velocities resulting from outbursts at a significant fraction of the Eddington limit. Table 1 presents a compilation of estimated jet Lorentz factors as a function of the X-ray luminosity at launch. In fact, with the exception of the upper limit for the LS sources (see below), these are all based upon observed proper motions in spatially resolved radio maps and are therefore only lower limits (see Fender 2003). These data are plotted in Fig 4 Note that a higher Lorentz factor in transient jets associated with outbursts is further supported by the much larger scatter in the $L_{\text {radio }}: L_{\mathrm{X}}$ plane compared to the LS sources (Gallo, Fender \& Pooley 2003).

The measured spread to the radio/X-ray correlation in LS black hole X-ray binaries was interpreted by Gallo, Fender \& Pooley (2003) in terms of a distribution in Doppler factors and used to infer an upper limit $\Gamma \lesssim 2$ to the bulk velocity of compact jets. The value of the spread as it appears in GFP03 is mainly determined by the two data sets for which the correlation extends over more than three orders of magnitude in X-ray luminosity, namely V 404 Cyg and GX 339-4. Obviously, errors in the distance estimates to these systems will introduce a further source of scatter to the correlation. While V 404 Cyg is (reasonably well) known to lie at $3.5 \mathrm{kpc}$ (Wagner et. al 1992), the distance to GX 339-4, and hence its luminosity, remain uncertain. Recent works (Hynes et al 2004; Zdziarski et al. 2004) have significantly revised the lower limit of $4 \mathrm{kpc}$ (Zdziarski et al. 1998) adopted in GFP03, placing GX 339-4 at a distance larger than $6 \mathrm{kpc}$. In particular, Hynes et al. (2004) discuss the possibility that the system could even be located on the far side of the Galaxy, at $\sim 15 \mathrm{kpc}$. In fact, any value between 6 and $15 \mathrm{kpc}$ has the effect of lowering the final spread to the radio/X-ray correlation by shifting GX 339-4 closer to V 404 Cyg, resulting in a more stringent upper limit on the jet bulk Lorentz factor. A minimum distance to GX 339-4 of $6 \mathrm{kpc}$ reduces the measured spread by a factor 1.6, requiring an outflow bulk velocity smaller than $0.7 c$. This sets a new upper limit of $\Gamma_{\text {radio }} \lesssim 1.4$ to the average bulk Lorentz factor of LS black hole X-ray binary jets; in fact, the distance to GX 339-4 would have to be larger than 15 $\mathrm{kpc}$ before the spread exceeds again the value obtained for $4 \mathrm{kpc}$. As discussed in GFP03, the above arguments are formally valid in case of radio beaming combined with isotropic X-ray emission, but if $\mathrm{X}$-rays are moderately beamed $\left(v_{X} \sim 0.3 \mathrm{c}\right.$, as suggested by e.g. models of dynamical coronae, Beloborodov 1999), the conclusions remain essentially unchanged.

However, Heinz \& Merloni (2004) argue that the spread

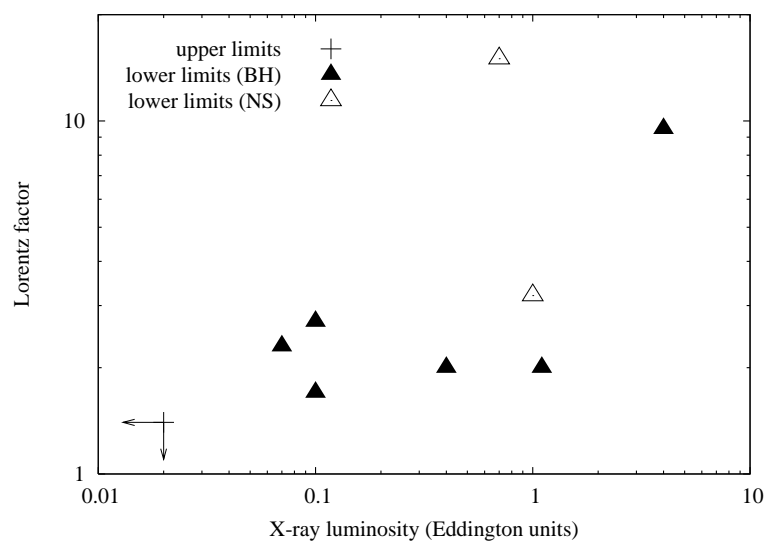

Figure 4. Limits on jet Lorentz factors as a function of the estimated bolometric X-ray luminosity at the time of jet launch. The arrows in the lower left of the figure indicate the condition that jets in the general LS state have $v \leq 0.8 c$. The rest of the symbols are lower limits only to the Lorentz factors from individual black hole (filled symbols) and neutron star (open symbols) X-ray binaries; the data are listed in table 1.

around the correlation can only really be used, in the absence of additional information, to constrain the range in jet velocities, and not the absolute values. Based upon their arguments, it remains likely that the bulk Lorentz factor of the LS jets is $\Gamma \sim 1$ but it is not a formal requirement. Based upon an analysis of the normalisations for GX 339-4 and V404 as estimated in Gallo, Fender \& Pooley (2003) they conclude that the Lorentz factors of the two sources differ by no more than a factor of two. Given the 'universality' within about one order of magnitude - of the correlation presented in Gallo, Fender \& Pooley (2003), this in turn implies that independent measurement of the Lorentz factor of a jet in the LS would apply to all LS sources. In the discussion that follows we shall continue to assume that the Lorentz factor of the steady jets $\Gamma \leq 2$ but note that it is not proven.

What is not clear from these data is whether the velocity is a simple 'step' function of the X-ray luminosity at launch, or rather a smoother function. As discussed in Fender (2003) a broad range in $\Gamma$ will, in most circumstances, produce approximately the same proper motions. For the simple unified model discussed in this article either ('step' or smooth function) interpretation is sufficient. The key factor is that the jets associated with the VHS/IS peak are probably more relativistic than those in the LS which generally precedes it.

\section{RADIO EMISSION AND JET POWER}

It is also crucial to estimate the jet power as a function of X-ray luminosity / state. In the following we present simplified expressions for the power in both optically thick and optically thin jets, in Eddington units, as a function of observable radio and X-ray emission.

\subsection{The low/hard state optically thick jet}

In Fender, Gallo \& Jonker (2003) it was argued that the total jet power $L_{\mathrm{J}}$, in the absence of significant advection, was related to the accretion luminosity $L_{\mathrm{X}}$ as follows:

$L_{\mathrm{J}}=A_{\text {steady }} L_{\mathrm{X}}^{0.5}$ 
where $A_{\text {steady }} \geq 6 \times 10^{-3}$ (the normalisation is referred to simply as $A$ in Fender, Gallo \& Jonker 2003).

Studies of the rapid variability from the 'hard' transient XTE $\mathrm{J} 1118+480$, which remained in the LS throughout its outburst, have supported the idea that the optical emission may originate in an outflow and not reprocessed emission from the disc (Merloni, di Matteo \& Fabian 2000; Kanbach et al. 2001; Spruit \& Kanbach 2002; Malzac et al. 2004). Detailed modelling of the correlated variability by Malzac, Merloni \& Fabian (2004) has resulted in a normalisation of the jet/outflow power which corresponds to $A_{\text {steady }} \sim 0.3$ in the above formalisation, which would imply that all LS sources are jet-dominated. For now we shall take this as the largest likely value of $A_{\text {steady }}$ (see also Yuan, Cui \& Narayan 2004 who estimate a value for the radiative efficiency for the jet in XTE J1118+480 which lies between the lower limit of Fender, Gallo \& Jonker 2003 and the estimate of Malzac, Merloni \& Fabian 2004).

\subsection{The optically thin jets}

The power associated with the production of optically thin jets can be calculated from the peak luminosity and rise time of the event, adapting the minimum energy arguments of Burbidge (1956, 1959), as follows:

$$
L_{\mathrm{J}}=20 \Delta t^{2 / 7} L_{\text {radio }}^{4 / 7} M^{-3 / 7}=2 \times 10^{-5} \Delta t^{2 / 7} d^{8 / 7} S_{5 \mathrm{GHz}}^{4 / 7} M^{-1}
$$

where $L_{\mathrm{J}}$ is the mean power into jet production (in Eddington units), $\Delta t$ is the rise time of the event, in seconds, $L_{\text {radio }}$ is the peak radio luminosity of the event at $5 \mathrm{GHz}$ (in Eddington units), $d$ is the distance in kpc, $\mathrm{S}_{5 \mathrm{GHz}}$ is the peak radio flux density at $5 \mathrm{GHz}$ (in mJy), and $M$ is the black hole mass in solar units. The equation assumes an emitting plasma with volume corresponding to $4 \pi(\Delta t c)^{3}$, a filling factor of unity, negligible energy in protons and a spectral index of $\alpha=-0.75$. See Longair (1994) for a fuller discussion.

In addition, since we have argued above that the bulk Lorentz factor is considerably higher for the transient jets underlying these optically thin outbursts, we need to compensate for the resultant Doppler effects. Fender (2001b) demonstrated that it is much more likely, for significantly relativistic jets, that the jet power is underestimated than overestimated, and introduced a correction factor $F(\Gamma, i)=\Gamma \delta^{-3}$ where $\delta$ is the relativistic Doppler factor associated with bulk Lorentz factor $\Gamma$ (the correction includes the kinetic energy of bulk flow). For $2 \leq \Gamma \leq 5$ the mean value of $F(\Gamma, i)$ averaged over $\cos (i)$ is $\sim 50$. We adopt this value as an additional (upward) correction to the power of the optically thin jets. Comparison of the formula given above with specific examples more carefully considered, e.g. the 1997 ejections from GRS 1915+105 reported by Fender et al. (1999), indicate this to be a reasonable correction. As discussed earlier, however, we have no clear upper limit on $\Gamma$ associated with these events, and the correction could be much larger. For example, for $2 \leq \Gamma \leq 7$ the mean value of $F(\Gamma, i)$ is $\sim 160$, and for $2 \leq \Gamma \leq 10$ is it $\sim 575$.

In table 1 we list in columns eight and nine the estimated optically thin radio powers during the flare events, $L_{\mathrm{J}}$, and the corresponding peak 'soft VHS/IS' X-ray luminosity, $L_{\mathrm{X}, \mathrm{VHS}}$, both expressed as Eddington fractions. These values are plotted against each other in Fig 5, and compared with the functions for the steady / LS jets as outlined above, for both the Fender, Gallo \& Jonker (2003) lower limit and the Malzac, Merloni \& Fabian (2004) estimate.

A best-fit power-law to the data for the transient events is of the form

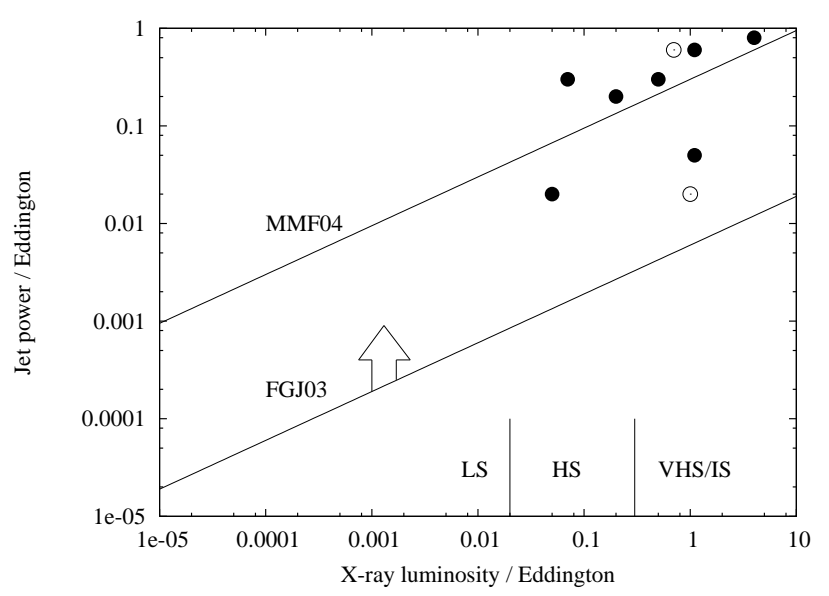

Figure 5. Comparing estimated jet power in the steady / LS and transients / VHS/IS states. Note that the uncertainties on the estimated jet powers are large, at least one order of magnitude. The solid line marked FGJ03 indicates the lower limit to the steady jet power in the LS as estimated by Fender, Gallo \& Jonker (2003). The line marked MMF04 indicates the same function with the larger normalisation as calculated by Malzac, Merloni \& Fabian (2004). The (solid) points indicate the estimates of jet power for the transient events listed in table 1 . It is interesting to note that the data are therefore compatible with the MMF04 relation at all X-ray luminosities. If the steady LS jet power is lower, nearer to the lower limit indicated by the FGJ03 function, then there may be a step up in jet power for the transient events. A very approximate indication of the regimes typically corresponding to the different X-ray states is indicated at the bottom of the figure. The power estimates for the two neutron star sources, Sco X-1 and Cir X-1, are indicated by open symbols.

$L_{\mathrm{jet}}=A_{\mathrm{trans}} L_{\mathrm{X}}^{0.5 \pm 0.2}$

where the fitted value is $A_{\text {trans }}=(0.4 \pm 0.1)$. Note that since for the transient jets $L_{\mathrm{X}} \sim 1$ this indicates near equipartition of $L_{\mathrm{X}}$ and $L_{\mathrm{J}}$ around the time of such events.

The index of the fit, $0.5 \pm 0.2$ is comparable to that derived for the LS, namely $L_{\text {jet }} \propto L_{\mathrm{X}}^{0.5}$ (Fender, Gallo \& Jonker 2003). The value of the normalisation, $A_{\text {trana }}$, is much larger than the conservative value for the steady jet normalisation $A_{\text {steady }}$ estimated in Fender, Gallo \& Jonker (2003; see above). However, it is only $\sim 50 \%$ larger than the value of $A_{\text {steady }}$ implied by the results of Malzac, Merloni \& Fabian (2004). Were such a large value to be valid for the LS it would imply that black hole X-ray binaries are likely to be jet-dominated below $L_{\mathrm{X}}=A_{\text {steady }}^{2} \sim 0.1 L_{\mathrm{Edd}}$. Since most sources do not strongly exceed this Eddington ratio while in a 'hard' X-ray state (see e.g. Figs 3 and 5), it implies that only in the HS and 'soft' VHS/IS and states, when the jet is suppressed, is the X-ray luminosity dominant over the jet (see also discussion in Malzac et al. 2004). Put another way, for such a large normalisation, whenever the jet is on, it is the dominant power output channel.

\subsection{Some caveats}

Note that there are very large uncertainties remaining in the estimation of both $A_{\text {steady }}$ and $A_{\text {trans. }}$. The functions used for the power of the steady jets are based upon essentially one detailed example XTE J1118+480 - and extrapolated to other sources via the 'universal' $L_{\text {radio }} \propto L_{\mathrm{X}}^{0.7}$ relation (although estimates from a handful of other sources are also compatible). While this approach may well be appropriate, spectral changes - in particular the location of the optically thin break in the synchrotron spectrum - could strongly 
affect the variation of $L_{\mathrm{J}}$ as a function of $L_{\mathrm{X}}$ and more work needs to be done in the future.

In the case of the power function for the transient jets, we have in fact more independent measurements, but those measurements themselves probably have a greater associated uncertainty. Underestimation of $L_{\mathrm{J}}$ can clearly arise due to deviations from equipartition, a lack of knowledge of the high-frequency extension of the synchrotron spectrum and a possible underestimate of the bulk Lorentz factor. Overestimation of $L_{r m J}$ could arise due to overestimation of the synchrotron-emitting volume (ie. a filling factor $f<1$ or injection/acceleration of particles into a confined jet).

\section{A single function or a step up in jet power?}

Nevertheless, it is noteable that a single power-law relation could be plotted through both the steady LS and transient VHS/IS functions, and that the normalisation of such a single function would be close to that estimated by Malzac, Merloni \& Fabian (2004), i.e. $A_{\text {steady }} \sim A_{\text {trans }} \sim 0.3$.

If there is not a single function then it seems that the transient VHS/IS jets are somewhat more powerful as a function of $L_{\mathrm{X}}$ than an extrapolation of the steady LS jets function. This suggestion is strengthened by our argument, below, that the optically thin events are likely to arise in internal shocks which do not dissipate $100 \%$ of the available kinetic energy. If real, this effect may be due to temporarily increased efficiency of jet production in the inner disc, or the transient addition of a new power source, namely the black hole spin (e.g. Blandford \& Znajek 1977; Punsly \& Corotoni 1991; Livio, Ogilvie \& Pringle 1999; Meier 1999, 2001, 2003; Koide et al. 2002). Nevertheless, we consider the similarity in both gradient and normalisation of the two jet functions to be remarkable. We note that for the model outlined in this paper to be tested against higher-mass (intermediate or supermassive) black holes, a further mass term would be required for both expressions (see Heinz \& Sunyaev 2003; Merloni, Heinz \& di Matteo 2003; Falcke, Körding \& Markoff 2004). However, for the X-ray binaries where the range in mass is likely to be $\leq 2$ this is not important at the current level of accuracy.

\section{INTERNAL SHOCKS}

The arguments given above clearly indicate that as the X-ray luminosity of the accreting source increases, then so does the velocity of the outflow (although whether this is in the form of a step, or other functional form, is as yet unclear). Since most, probably all, outbursting sources have followed a path in which they have become monotonically brighter in a hard state before making a transition to a soft state, this tells us that a shock should form in the previously-generated 'steady' jet as the faster-moving VHS/IS jet catches up and interacts with it. This internal shock is therefore a natural origin for the optically thin events observed at the beginning of X-ray transient outbursts. Internal shocks have previously been proposed for AGN (e.g. Rees 1978; Marscher \& Gear 1985; Ghisellini 1999; Spada et al. 2001) and gamma-ray bursts (GRBs) (e.g. Rees \& Meszaros 1994; van Paradijs, Kouveliotou \& Wijers 2000 and references therein). Indeed in the context of X-ray binaries an internal-shock scenario has already been discussed previously for GRS $1915+105$ by Kaiser, Sunyaev \& Spruit(2000), Vadawale et al. (2003) and Turler et al. (2004), and their ideas have significantly inspired this work.

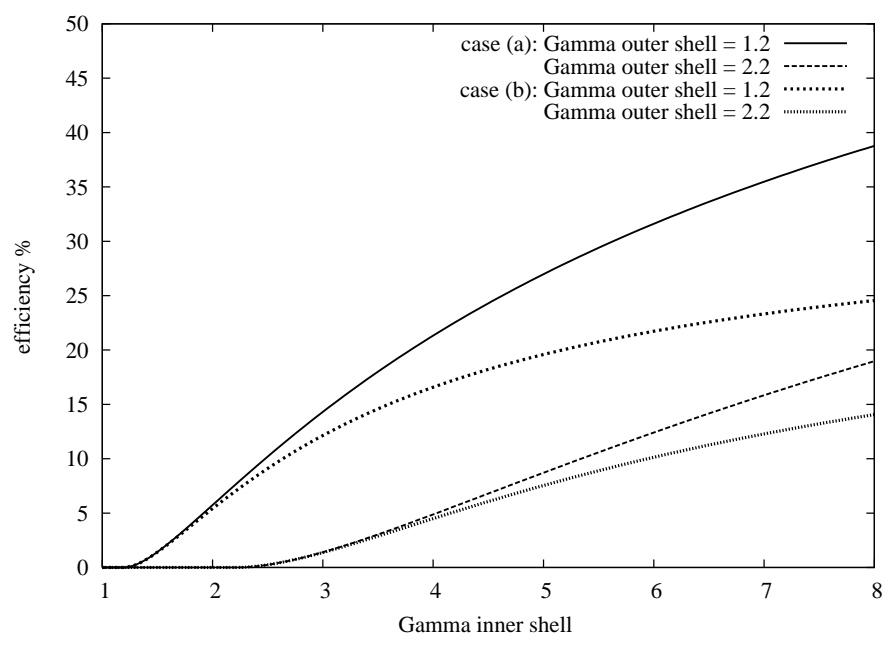

Figure 6. Efficiency of energy release for the collision of two shells, where the outer shell is only mildly relativistic (bulk Lorentz factor [BLF] $\Gamma<2$, under the assumption of conservation of momentum and energy. Case (a) corresponds to the case of two blobs with equal total energy (i.e. $\left.\Gamma_{1} M_{1}=\Gamma_{2} M_{2}\right)$ in which case the jet power has not increased. Case (b) corresponds to the case of the same mass but increased Lorentz factor, ie. an increase in jet power by a factor $\Gamma_{2}-\Gamma_{1}$. This can be considered a (high oversimplified) approximation to the collision of a relativistic VHS/IS jet (inner shell) with the preceding steady LS jet (outer shell).

Rees \& Meszaros (1994) spelled out the basis for such 'internal shocks' in the context of GRBs. They assumed two 'blobs' of equal mass but differing Lorentz factors were ejected such that the later ejection had the higher Lorentz factor. This component, if moving along precisely the same trajectory as the original component, will collide with it. Assuming conservation of energy and momentum it was shown that up to $40 \%$ of the total kinetic energy could be released in this shock.

The formulation for the efficiency of energy release, $\epsilon$, as presented in Rees \& Meszaros corresponds to the case in which both blobs have Lorentz factors $\Gamma_{1,2}>>1$. We have repeated their approach, but considered instead the (less simple) case in which the first blob is at most only mildly relativistic $\left(\Gamma_{1}<2\right)$.

In Fig 7 we plot the internal shock efficiency for two cases:

- (a) The blobs have the same total energy, fulfilling the criterion that $\Gamma_{1} M_{1}=\Gamma_{2} M_{2}$ (where $M$ is the mass of the blob). This corresponds to the situation in which the jet power does not increase significantly, while the Lorentz factor does.

- (b) The blobs have equal mass. This corresponds to a genuine increase in jet power by a factor $\Gamma_{2}-\Gamma_{1} \sim \Gamma_{2}$. This corresponds to the maximum efficiency for the internal shock (e.g. Spada et al. 2001).

For the kind of values estimated for transient jets, efficiencies in the range $5-40 \%$ are expected. For a total jet power of $P_{\mathrm{J}}$, the power in the shock (i.e. available for particle acceleration) will be $P_{\text {shock }} \leq \epsilon P_{\mathrm{J}}$. The remaining jet power $(\epsilon-1) P_{\mathrm{J}}$ is associated with the kinetic energy of the merged shells and should eventually be dissipated via interactions with the ambient medium.

Of course this is a highly oversimplified approximation of the true circumstances as the jet increases in velocity. Nevertheless, it illustrates that the internal shock produced by a transient (as it is subsequently shut down in the soft state) acceleration of the jet from $\Gamma \sim 1$ to $\Gamma \lesssim 10$ could produce dissipation in an internal shock with an observed energy release comparable (within the or- 
der or magnitude uncertainty) to that estimated for the steady jet prior to the acceleration. If, as seems likely, $A_{\text {trans }} \geq A_{\text {steady }}$ then the more efficient shock scenario (b) (Fig 7) is more likely, and the total jet power, and not just velocity, has significantly increased. Beloborodov (2000) discusses in further detail the high radiative efficiencies which may be obtained in the internal shock model.

The internal shock scenario is also attractive as an explanation for why the same radio flux at a given radio frequency for a given source can be sometimes optically thin and sometimes optically thick. Consider GRS 1915+105, where in the plateau states a flux density of $\sim 40 \mathrm{mJy}$ at $15 \mathrm{GHz}$ may be associated with an optically thick spectrum, and later a comparable flux density with optically thin rising phases of oscillation events (e.g. Fender et al. 1999). If the particle acceleration all occurred at the base of a jet with an approximately fixed structure, this is hard to explain. However, it follows naturally from a scenario where the optically thin events are associated with internal shocks occurring at a much larger distance from the dense inner jet.

Note that it is the radiation resulting from the energy liberated by the internal shock, which we have measured in order to estimate the jet power in section 5 above. However, since our estimates of the bulk Lorentz factor must be based upon observations of the post-shock plasma, then the true jet power must be larger by a factor $\epsilon^{-1}$. Since $0.05 \lesssim \epsilon \lesssim 0.45$ in the above simplification, this may imply that the underlying jet power is actually a further order of magnitude larger for the transient jets. In this case a single function corresponding to both the LS and VHS/IS jets seems less likely.

As discussed in Vadawale et al. (2003) the strength of the shock is likely to be related to the amount of material lying in the path of the faster 'VHS/IS' jet. They discussed this in the context of GRS 1915+105, where the strength of 'post-plateau jets' (KleinWolt et al. 2002) is shown to be correlated with the total X-ray fluence of the preceding 'plateau' (which was presumably a phase of slower jet production). Generalising this phenomenon to other Xray transients, it provides a natural explanation for why, although there are often multiple radio flaring events, the first is invariably the strongest.

\section{TOWARDS A UNIFIED MODEL}

Based upon the key generic observational details assembled above, we have attempted to construct a unified, semi-quantitative, model for the disc-jet coupling in black hole X-ray binaries. A simplified version of the model specific to GRS 1915+105 has been presented in Fender \& Belloni (2004). The model is summarised in Fig 7, which we describe in detail below. The diagram consists of a schematic X-ray hardness-intensity diagram (HID) above a schematic indicating the bulk Lorentz factor of the jet and inner accretion disc radius as a function of X-ray hardness. The four sketches around the outside of the schematics indicate our suggestions as to the state of the source at the various phases $\mathbf{i}-\mathbf{i v}$. The path of a typical X-ray transient is as indicated by the solid arrows.

- Phase i: Sources are in the low-luminosity LS, producing a steady jet whose power correlates as $L_{\text {jet }} \propto L_{\mathrm{X}}^{0.5}$ (ignoring any mass term). This phase probably extends down to very low luminosities ('quiescence').

- Phase ii: The motion in the HID, for a typical outburst, has been nearly vertical. There is a peak in the LS after which the motion in the HID becomes more horizontal (to the left) and the source moves into the 'hard' VHS/IS. Despite this softening of the X-ray spectrum the steady jet persists, with a very similar coupling, quantitatively, to that seen in the LS.

- Phase iii: The source approaches the 'jet line' (the solid vertical line in the schematic HID) in the HID between jet-producing and jet-free states. As the boundary is approached the jet properties change, most notably its velocity. The final, most powerful, jet, has the highest Lorentz factor, causing the propagation of an internal shock through the slower-moving outflow in front of it.

- Phase iv: The source is in the 'soft' VHS/IS or the canonical HS, and no jet is produced. For a while following the peak of phase iii fading optically thin emission is observed from the optically thin shock.

Following phase iv, most sources drop in intensity in the canonical HS until a (horizontal) transition back, via the VHS/IS, to the LS. Some sources will make repeated excursions, such as the loops and branches indicated with dashed lines in Fig 7, back across the jet line, However, with the exception of GRS 1915+105, the number of such excursions is generally $\leq 10$. When crossing the jet line from right to left, the jet is re-activated but there is (generally) no slower-moving jet in front of it for a shock to be formed; only motion from left to right produces an optically thin flare (this is a prediction). Subsequently the motion back towards quiescence is almost vertically downwards in the HID.

The model as outlined above has many similarities with the scenarios described by Meier $(1999,2001,2003)$ who has approached the problem from a more theoretical point of view. Meier (2001) has suggested that in low-luminosity states the jet is powered by a modification of the Blandford \& Payne ('BP') (1982) mechanism taking into account frame-dragging near a rotating black hole (Punsly \& Coroniti 1990). This 'BP/PC mechanism' can extract black hole spin by the coupling of magnetic field lines extending from within the ergosphere to outside of it. Meier (2001) further suggests that during phases of very high accretion the Blandford \& Znajek ('BZ') (1977) mechanism may work briefly. This may be associated with a 'spine jet' which is considerably more relativistic than the 'sheath jet' produced by the BP/PC mechanism. Note that the power of the jets as given in Meier (2001, 2003 ) is about linearly proportional to the accretion rate; in the formulation of Fender, Gallo \& Jonker (2003) this corresponds to the 'jet dominated state' (see also Falcke, Kording \& Markoff 2004).

We can revisit the scenarios of Meier in the light of our compilation of observational results and steps toward a unified model. In the faint LS (phase $\mathbf{i}$ in Fig 7) is the jet formed by the BP or BP/PC mechanisms ? Given that the jet may be formed at relatively large distances from the black hole in such states, there may not be any significant influence of the black hole spin on the jet formation process. However, it is also likely that in such states the jet-formation process is not occurring within thin discs, as is the basis of the BP mechanism, but rather in a geometrically thick flow (see also e.g. Blandford \& Begelman 1999; Meier 2001; Merloni \& Fabian 2002)

As the accretion rate increases the power of this disc-jet will increase and the geometrically thin accretion disc will propagate inwards. During this phase the jet formation process may migrate from $\mathrm{BP} \rightarrow \mathrm{BP} / \mathrm{PC}$. However, the suggestion that the most relativistic jets are formed by the BZ process seems at odds with the observation of significantly relativistic outflows from two neutron stars systems (Fomalont et al. 2001a,b; Fender et al. 2004). In a related work, the results of Yu, van der Klis \& Fender (2004) indicate that the subsequent evolution of X-ray transient outbursts is approximately determined before the soft VHS/IS peak, in both neutron star and black hole systems. This suggests that already by the time 


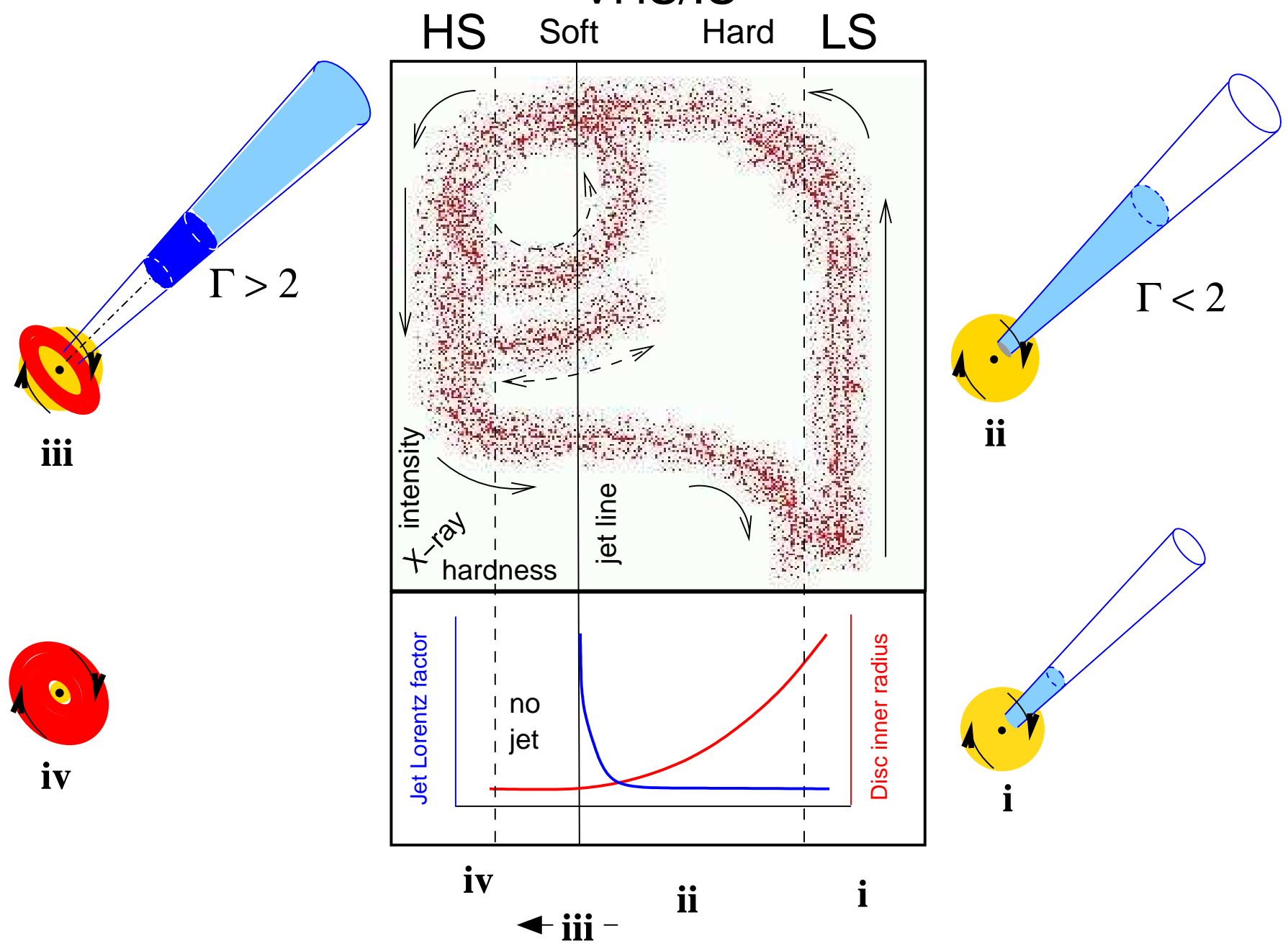

Figure 7. A schematic of our simplified model for the jet-disc coupling in black hole binaries. The central box panel represents an X-ray hardness-intensity diagram (HID); 'HS' indicates the 'high/soft state', 'VHS/IS' indicates the 'very high/intermediate state' and 'LS' the 'low/hard state'. In this diagram, X-ray hardness increases to the right and intensity upwards. The lower panel indicates the variation of the bulk Lorentz factor of the outflow with hardness - in the LS and hard-VHS/IS the jet is steady with an almost constant bulk Lorentz factor $\Gamma<2$, progressing from state $\mathbf{i}$ to state ii as the luminosity increases. At some point - usually corresponding to the peak of the VHS/IS $-\Gamma$ increases rapidly producing an internal shock in the outflow (iii) followed in general by cessation of jet production in a disc-dominated HS (iv). At this stage fading optically thin radio emission is only associated with a jet/shock which is now physically decoupled from the central engine. As a result the solid arrows indicate the track of a simple X-ray transient outburst with a single optically thin jet production episode. The dashed loop and dotted track indicate the paths that GRS $1915+105$ and some other transients take in repeatedly hardening and then crossing zone iii - the 'jet line' - from left to right, producing further optically thin radio outbursts. Sketches around the outside illustrate our concept of the relative contributions of jet (blue), 'corona' (yellow) and accretion disc (red) at these different stages.

of the LS peak we can estimated the size of the ejection even which is to follow, and is a further indication that the study of neutron stars will shed important light on the physics of jet formation in black hole systems.

\subsection{Ejected disc or ejected corona?}

It is interesting to compare the sequence of events we have outlined as being responsible for ejection events with the interpretation most commonly put forward when the disc-jet coupling in GRS $1915+105$ was first observed. In this source oscillations, on timescales of tens of minutes, between hard (state ' $\mathrm{C}$ ' $\equiv$ hard VHS/IS) and soft (states 'A' and 'B' $\equiv$ soft VHS/IS - see Fig 1(a)) were associated with cycles of disc 'emptying' and refill (e.g. Bel- loni et al. 1997b). When the relation to ejection events (Pooley \& Fender 1997; Eikenberry et al. 1998; Mirabel et al. 1998) was discovered, it was suggested that the 'disappearance' of the inner disc was directly related to its (partial) ejection (see also Feroci et al. 1999; Nandi et al. 2001). The following sequence of events was envisaged:

(i) Thin disc extends close to black hole (soft state)

(ii) Inner disc is ejected, resulting in:

- Disappearance of inner disc $\rightarrow$ transition to hard state

- Synchrotron event

(iii) Refill of disc $\rightarrow$ return to soft state

However, Vadawale et al. (2003) argued that it was the 'corona' which was subsequently ejected as the disc moved in, 
again specifically for the case of GRS 1915+105. Rodriguez, Corbel \& Tomsick (2003) have also suggested, in the case of XTE $\mathrm{J} 1550-564$, that it is coronal, and not disc, material which is ejected prior to radio outburst

It is clear that if the model we have outlined in this paper is correct, the 'disc-ejection' scenario is unlikely to be, for any black hole X-ray binary. Specifically, it is the transition towards the soft state (that is, the 'refill' of the inner disc) which causes the ejection event. Therefore, if we are to consider the ejection of mass, it is more likely the 'corona' (or whatever form the accretion flow has in the harder states) and not the 'disc' which is ejected.

\section{CONCLUSIONS}

We have examined the observational properties of the jets associated with black hole X-ray binary systems. The key observations can be summarised as:

(i) The radio:X-ray coupling: we have established that the steady radio emission associated with the canonical LS persists beyond the softening of the X-ray spectrum in the 'hard' VHS/IS. At the end of the transtion from 'hard' to 'soft' VHS/IS, usually associated with a local maximum in the $\mathrm{X}$-ray light curve, a transient radio outburst occurs. The radio emission is subsequently suppressed until the source X-ray spectrum hardens once more. Some source may repeatedly make the transition from 'hard' to 'soft' VHS/IS and back again, undergoing repeated episodes of steady and transient jet formation.

(ii) Jet velocities: we have argued that the measured velocities for the transient jets, being relativistic with $\Gamma \gtrsim 2$ are significantly larger than those of the steady jets in the LS, which probably have $\Gamma \lesssim 1.4$.

(iii) Jet power: we have furthermore established that our best estimates of the power associated with the transient jets are compatible with extrpolations of the functions used to estimate the power in the LS (albeit with a relatively large normalisation).

Essentially equivalent conclusions about the radio:X-ray coupling have been drawn by Corbel et al. (2004). Putting these observational aspects together we have arrived at a semi-quantitative model for jet production in black hole XRBs. We argue that for Xray spectra harder than some value (which may be universal or vary slightly from source to source) a steady jet is produced. The power of this jet correlates in a non-linear way (approximately given as $L_{\mathrm{J}} \propto L_{\mathrm{X}}^{0.5}$ ) with the X-ray luminosity. As the X-ray luminosity increases above $\sim 1 \%$ of the Eddington rate the $\mathrm{X}$-ray spectrum begins to soften. Physically this probably corresponds to the heating of the inner edge of the accretion disc as it propagates inwards with increasing accretion rate. Initially the jet production is not affected. As the disc progresses inwards the jet velocity increases. As it moves through the last few gravitational radii before the ISCO, the Lorentz factor of the jet rises sharply, before the jet is suppressed in a soft disc-dominated state. The rapid increase in jet velocity in the final moments of its existence results in a powerful, optically thin, internal shock in the previously existing slower moving outflow.

The inner disc may subsequently recede, in which case a steady jet is reformed, but with decreasing velocity and therefore no internal shocks. If the disc once more moves inwards and reaches the 'fast jet' zone, then once more an internal shock is formed. In fact while jets are generally considered as 'symptoms' of the underlying accretion flow, we consider it possible that the reverse may be true. For example, it may be the 'growth' of the steady jet (via e.g. build up of magnetic field near the ISCO / black hole) which results in the hardening of the X-ray spectrum, perhaps via pressure it exerts on the disc to push it back, or simply via Comptonisation of the inner disc as it spreads (for further discussions see e.g. Nandi et al. 2001; Tagger et al. 2004).

In the context of the nature and classification of black hole 'states', these states, whether 'classical' or as redefined by McClintock \& Remillard (2004) do not have a one-to-one relation with the radio properties of the source. It seems that as far as the jet is concerned, it is 'on' - albeit with a varying velocity - if the disc does not reach 'all the way in', which probably means as far as the ISCO. The dividing 'jet line' (Fig 8) HID, may also correspond, at least approximately, to a singular switch in X-ray timing properties (Belloni et al. 2004; Homan \& Belloni 2004; see also once more discussion in McClintock \& Remillard 2004) and may be the single most important transition in the accretion process. Further study of the uniqueness of the spectral and variability properties of sources at this transition point should be undertaken to test and refine our model.

Finally, given that Merloni, Heinz \& di Matteo (2003) and Falcke, Körding \& Markoff (2004) (see also Heinz \& Sunyaev 2003; Maccarone, Gallo \& Fender 2003) have recently demonstrated quantitatively the scaling of radio:X-ray coupling across a range of $\gtrsim 10^{7}$ in black hole mass, it is obviously of great interest to see if the model we are working towards for the coupling of accretion and jet formation in black hole binaries may also be applied to AGN. In addition, detailed modelling of the internal shock scenario is required to see if the coupling, as outlined above, really could allow us to predict radio light curves from X-ray, and vice versa. These two areas should be the next steps forward.

\section{ACKNOWLEDGEMENTS}

RPF would like to thank many people for useful discussions related to the ideas presented here, including Catherine Brocksopp, Annalisa Celotti, Stephane Corbel, Jeroen Homan, Peter Jonker, Marc Klein-Wolt, Tom Maccarone, Dave Meier, Simone Migliari, Jon Miller and Felix Mirabel. We thank the referee, Andrea Merloni, for thoughtful and detailed comments on the paper.

\section{REFERENCES}

Beloborodov A. M., 1999, ApJ, 510, 123

Beloborodov A. M., 2000, ApJ, 539, L25

Belloni T., 2004, to appear in Proc. of the II BeppoSAX Meeting 'The restless High-Energy Universe', Eds. van den Heuvel E.P.J., in 't Zand J.J.M. and Wijers R.A.M.J. astro-ph/0309028,

Belloni T., Mendez M., van der Klis M., Hasinger G., Lewin W.H.G., van Paradijs J., 1996, ApJ, 472, L107

Belloni T., Mendez M., King A.R., van der Klis M., van Paradijs J., 1997, ApJ, 488, L109

Belloni T., Klein-Wolt M., Mendez M., van der Klis M., van Paradijs J., 2000, A\&A, 355, 271

Belloni, T., Homan, J., Nespoli, E., Casella, P., van der Klis, M., Lewin, W.H.G., Miller, J.M., Méndez, M., 2004, A\&A, submitted

Blandford R., Znajek R.L., 1977, MNRAS, 179, 433

Blandford R., Königl A., 1979, ApJ, 232, 34

Blandford R.D., Begelman M.C., 1999, MNRAS, 303, L1

Brocksopp C. et al., 2002, 331, 765

Burbidge G.R., 1956, Phys. Rev., 103, 264

Burbidge G.R., 1959, ApJ, 129, 849 
Corbel S., Fender R.P., 2002, ApJ, 573, L35

Corbel S., Fender R.P., Tzioumis A.K., Nowak M., McIntyre V., Durouchoux P., Sood R., 2000, A\&A, 359, 251

Corbel S. et al., 2001, ApJ, 554, 43

Corbel S., Fender R.P., Tzioumis A.K., Tomsick J.A., Orosz J.A., Miller J.M., Wijnands R., Kaaret P., 2002, Science, 298, 196

Corbel S., Nowak M.A., Fender R.P., Tzioumis A.K., Markoff S., 2003, A\&A, 400, 1007

Corbel S., Fender R., Tomsick J.A., Tzioumis A.K., 2004, ApJ, submitted

Dhawan V., Mirabel I.F., Rodríguez L.F., 2000, ApJ, 543, 373

Esin A.E., McClintock J.E., Narayan R., 1997, ApJ, 489, 865

Falcke H., Biermann P.L., 1996, A\&A, 308, 321

Falcke H., Körding E., Markoff S., 2004, A\&A, 414, 895

Fender R.P., 2001a, Black Holes in Binaries and Galactic Nuclei. Proceedings of the ESO Workshop held at Garching, Germany, 6-8 September 1999. Lex Kaper, Edward P. J. van den Heuvel, Patrick A. Woudt (eds.), p. 193. Springer.

Fender R.P., 2001b, MNRAS, 322, 31

Fender R.P., 2003, MNRAS, 340, 1353

Fender R.P., 2004, in 'Compact Stellar X-ray Sources', eds. W.H.G. Lewin and $\mathrm{M}$. van der Klis, in press, astro-ph/0303339,

Fender R., Belloni T., 2004, ARA\&A, 42, 317

Fender R.P., Gallo E., Jonker P., 2003, MNRAS, 343, L99

Fender R.P., Garrington S.T., McKay D.J., Muxlow T.W.B., Pooley G.G., Spencer R.E., Stirling A.M., Waltman E.B., 1999a, MNRAS, 304, 865

Fender R. et al., 1999b, ApJ, 519, L165

Fender R., Wu K., Johnston H., Tzioumis T., Jonker P., Spencer R., van der Klis M., 2004, Nature, 427, 222

Feroci M., Matt G., Pooley G., Costa E., Tavani M., Belloni T., 1999, A\&A, 351, 985

Fomalont E.B., Geldzahler B.J., Bradshaw C.F., 2001a, ApJ, 553, L27

Fomalont E.B., Geldzahler B.J., Bradshaw C.F., 2001b, ApJ, 558, 283

Fuchs Y. et al., 2003, A\&A, 409, L35

Gallo E., Fender R.P., Pooley G.G., 2003, MNRAS, 344, 60

Gallo E., Corbel S., Fender R.P., Maccarone T.J., Tzioumis A.K., 2004, MNRAS, 347, L52

Gallo E., Fender R.P., Hynes R.I., 2004, MNRAS, submitted

Ghisellini G., 1999, Astronomische Nachrichten, 320, 232

Hannikainen D.C., Campbell-Wilson D., Hunstead R., McIntyre V., Lovell J., Reynolds J., Tzioumis T., Wu, K., 2001, ApSSS, 276, 45

Hannikainen D.C. et al., 2004, in prep

Harmon B.A. et al., 1995, Nature, 374, 703

Harmon B.A., Deal K.J., Paciesas W.S., Zhang S.N., Robinson C.R., Gerard E., Rodríguez L.F., Mirabel I.F., 1997, ApJ, 477, L85

Heinz S., Sunyaev R.A., 2003, MNRAS, 343, L59

Heinz S., Merloni A., 2004, MNRAS, submitted

Hjellming R.M., Johnston K.J., 1988, ApJ, 328, 600

Hjellming R.M., Rupen M.P., 1995, Nature, 375, 464

Hjellming R.M., Rupen M.P., Mioduszewski A.J., Smith D.A., Harmon B.A., Waltman E.B., Ghigo F.D., Pooley G.G., 1998, BAAS, 30, 1405

Homan J., Belloni T., 2004, in prep

Homan J., Wijnands R., van der Klis M., Belloni T., van Paradijs J., KleinWolt M., Fender R., Mèndez M., 2001, ApJS, 132, 377

Hynes R.I., Haswell C.A., Chaty S., Shrader C.R., Cui W., 2002, MNRAS, 331, 169

Hynes R. I., Steeghs D., Casares J., Charles P. A., O’Brien K., 2004, ApJ, in press astro-ph/0402408,

Jonker P.G., Gallo E., Dhawan V., Rupen M., Fender R.P., Dubus G., 2004, MNRAS, 351, 1359

Kaiser C.R., Sunyaev R., Spruit H.C., 2000, A\&A, 356, 975

Kanbach G., Straubmeier C., Spruit H.C., Belloni T., 2001, Nature, 414, 180

Klein-Wolt M., Fender R.P., Pooley G.G., Belloni T., Migliari S., Morgan E.H., van der Klis M., 2002, MNRAS, 331, 745

Koide S., Shibata K., Kudoh T., Meier D.L., 2002, Science, 295, 1688

Levine A.M., Bradt H., Cui W., Jernigan J.G., Morgan E.H., Remillard R.A., Shirey R., Smith D., 1996, ApJ, 469, L33

Livio M., Ogilvie G.I., Pringle J.E., 1999, ApJ, 512, 100
McClintock J.E., Remillard R.A., 2004, in 'Compact Stellar X-ray Sources', eds. W.H.G. Lewin and M. van der Klis, in press, astro-ph/0306213

Maccarone T., 2003, A\&A, 409, 697

Maccarone T.J., Coppi P.S., 2003, MNRAS, 338, 189

Maccarone T., Gallo E., Fender R.P., 2003, 345, L19

Malzac J., Merloni A., Fabian A.C., 2004, MNRAS, 351, 253

Malzac J., Belloni T., Spruit H.C., Kanbach G., 2003, A\&A, 407, 335

Markwardt C., 2001, ApSSS, 276, 209

Markwardt, C.B., Marshall, F.E., Swank, J.H., 1999, IAUC, 7274

Marscher A.P., Gear W.K., 1985, ApJ, 298, 114

Marscher A.P., Jorstad S.G., Gomez J-L., Aller M.F., Terasranta H., Lister M.L., Stirling A.M., 2002, Nature, 417, 625

Meier D.L., 1999, ApJ, 522, 753

Meier D.L., 2001, ApJ, 548, L9

Meier D.L., 2003, New Astronomy Reviews, 47, 667

Meier D.L., Koide S., Uchida Y., 2001, Science, 291, 84

Merloni A., Fabian A.C., 2002, 332, 165

Merloni A., Fabian A.C., Ross R.R., 2000, MNRAS, 313, 193

Merloni A., Di Matteo T., Fabian A.C., 2000, MNRAS, 318, L15

Merloni A., Heinz S., di Matteo T., 2003, MNRAS, 345, 1057

Miller J.M. et al., 2002, ApJ, 578, 348

Mirabel, I.F., Rodríguez, L.F, 1994, Nature, 371, 46

Mirabel, I.F., Rodríguez, L.F, 1999, ARA\&A, 37, 409

Nandi A., Chakrabarti S.K., Vadawale S.V., Rao A.R., 2001, A\&A, 380, 245

Nespoli E., 2003, MSc thesis, Milan

Orosz J.A. et al., 2001, ApJ, 555, 489

Paredes J.M., Marti J., Peracaula M., Pooley G., Mirabel I.F., 2000, A\&A, 357,507

Pooley G.G., 2004, ATel \# 242

Punsly B., Coroniti F.V., 1990, ApJ, 354, 583

Rees M.J., 1978, MNRAS, 184, 61

Rees M.J., Meszaros P., 1994, ApJ, 430, L93

Reig P., Belloni T., van der Klis M., 2003, A\&A, 412, 229

Remillard, R.A., Sobczak, G.J., Muno, M.P., McClintock, J.E., 2002, 564, 962

Rodriguez J., Corbel S., Tomsick J.A., 2003, ApJ, 595, 1032

Sobczak G.J., McClintock J.E., Remillard R.A., Cui W., Levine A.M., Morgan E.H., Orosz J.A., Bailyn C.D., 2000, ApJ, 533, 993

Spada M., Ghisellini G., Lazzati D., Celotti A., 2001, MNRAS, 325, 1559

Spruit H.C., Kanbach G., 2002, A\&A, 391, 225

Stirling A.M., Spencer R.E., de la Force C.J., Garrett M.A., Fender R.P., Ogley R.N., 2001, MNRAS, 327, 1273

Tagger M., Varniere P., Rodriguez J., Pellat R., 2004, ApJ, 607, 410

Tanabaum H., Gursky H., Kellogg E., Giacconi R., Jones C., 1972, ApJ, 177, L5

Turler M., Courvoisier T.J.-L., Chaty S., Fuchs Y., 2004, A\&A, 415, L35

Vadawale S.V., Rao A.R., Naik S., Yadav J.S., Ishwara-Chandra C.H., Pramesh Rao A., Pooley G.G., 2003, ApJ, 597, 1023

Van Paradijs J., Kouveliotou C., Wijers R.A.M.J., 2000, ARA\&A, 38, 379

Wagner R. M., Kreidl T. J., Howell S. B., Starrfield S. G., 1992, ApJ, 401, L97

Wang X.Y., Dai Z.G., Lu T., 2003, ApJ, 592, 347

Wood, A., Smith, D.A., Marhsall, F.E., Swank, J.H., 1999, IAUC 7274

Wu K., Soria R., Campbell-Wilson D., Hannikainen D., Harmon B.A., Hunstead R., Johnston H., McCollough M., McIntyre V., 2002, ApJ, 565, 1161

Yu W., Klein-Wolt M., Fender R., van der Klis M., 2003, ApJ, 589, L33

Yu W., van der Klis M., Fender R., 2004, ApJ, 611, L121

Yuan F., Cui W., Narayan R., 2004, ApJ, submitted astro-ph/0407612

Zdziarski A. A., Poutanen J., Mikolajewska J., Gierlinski M., Ebisawa K., Johnson W. N., 1998, MNRAS, 301, 435

Zdziarski A. A., Gierlinski M., Mikolajewska J., Wardzinski G., Smith D. M., Harmon A. B., Kitamoto S., 2004, MNRAS, in press astro-ph/0402380 